Commun: Communication et organisation

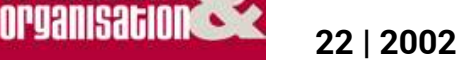

Interculturel et communication dans les organisations

\title{
Les échelles d'observation de la culture
}

\section{Dominique Desjeux}

\section{OpenEdition}

Journals

Édition électronique

URL : http://journals.openedition.org/communicationorganisation/2728

DOI : 10.4000/communicationorganisation.2728

ISSN : $1775-3546$

Éditeur

Presses universitaires de Bordeaux

Édition imprimée

Date de publication : 1 novembre 2002

ISSN : 1168-5549

\section{Référence électronique}

Dominique Desjeux, "Les échelles d'observation de la culture », Communication et organisation [En ligne], 22 | 2002, mis en ligne le 27 mars 2012, consulté le 01 mai 2019. URL : http://

journals.openedition.org/communicationorganisation/2728; DOI : 10.4000/

communicationorganisation. 2728

Ce document a été généré automatiquement le 1 mai 2019.

(c) Presses universitaires de Bordeaux 


\title{
Les échelles d'observation de la culture
}

\author{
Dominique Desjeux
}

\section{Introduction}

1 Quand un ingénieur d'une grande école parisienne m'explique, lors de mon premier séjour de 3 mois en Chine en 1997 qu'il existe une particularité chinoise qui est l'existence de réseaux, les fameux guanxi, je reçois cette information avec beaucoup d'étonnement : si un pays fonctionne bien sur la base de réseaux, c'est la France. Je pense tout particulièrement aux réseaux des grandes écoles et notamment celui du corps des mines sur lequel j'ai travaillé avec Michel Crozier, Erhard Friedberg et Jean Pierre Worms en 1969. Ce réseau est formé par les douze premiers sortant de l'école Polytechnique. Ce réseau favorise les liens entre le ministère de l'Industrie, les cabinets de la Présidence de la République, du Premier ministre et du ministre des Finances, et les entreprises des métaux non ferreux, nucléaires et pétrolières. Je ne vois donc pas en quoi l'existence de réseaux serait une particularité culturelle chinoise.

2 Après réflexion, je comprends que cet ingénieur français a des problèmes de lobbying en Chine et qu'il regrette sans s'en rendre compte son réseau d'anciens dont il avait incorporé le fonctionnement au point d'en oublier son existence. J'en ai tiré une grande règle: quand quelqu'un attribue la source d'un problème de management ou de vie quotidienne à l'autre culture, il m'informe d'abord sur sa propre culture et sur ce qui est important pour lui. Il ne m'apprend pas grand-chose sur les chinois, les américains, les africains ou les français. L'explication par la culture c'est dans un premier temps, celui de la découverte, un test projectif pour celui qui la mobilise. Elle apprend surtout sur la culture qui émet une appréciation sur l'autre.

3 Nous l'avons encore vérifié avec mon collègue et ami Zheng Li hua, en travaillant sur des publicités de vêtements françaises, allemandes et anglaises qui était montrées à des étudiants chinois dans le cadre d'une enquête sur l'image que les chinois se font des européens (Zheng Li hua, Dominique Desjeux, éds., 2002; 2003, PUF). La publicité 
française montrait un couple peu habillé et plutôt « collé ». Dans la publicité allemande le couple était un peu plus habillé, l'homme et la femme étant moins rapprochés. La publicité anglaise montrait un couple en tailleur et imperméable mastic, l'homme regardant dans une direction, et la femme dans une autre comme un vieux couple blasé, au moins d'un point de vue projectif occidental ! Pour les étudiants, la publicité française montrait un couple d'amants, l'allemande montrait un couple marie et la publicité anglaise un couple de fiancés...

4 Ici, les publicités européennes ont servi de révélateur aux normes chinoises exprimant les codes qui définissent ce qui relève de l'intime, du privé et du public. En effet, encore aujourd'hui à Guangzhou, même si c'est en train d'évoluer en milieu urbain et universitaire, il n'est pas considéré comme convenable d'exprimer en public l'intimité de l'amour pour un couple, surtout s'il est fiancé. Il faut montrer son " sérieux ». Le sérieux s'exprime grâce à une distance corporelle importante. Ceci ne veut pas non plus dire que ce code relève d'une essence hors de l'histoire, qu'il perdure depuis des siècles ou qu'il perdurera encore longtemps. Il est déjà en train de changer. La deuxième leçon est que la culture n'est pas une essence immuable. La culture est une dynamique encastrée dans une durée historique plus ou moins longue.

\section{Les échelles d'observation de la culture : une prise de conscience des limites de la connaissance}

5 La culture est à la fois une structure et une dynamique, c'est ce qui rend son analyse et son observation si difficile. Comme structure elle comprend des éléments stables qui peuvent donner l'impression d'une essence alors que cette stabilité relève surtout de la longue durée historique. Comme dynamique elle est soumise au changement et à l'histoire. En fonction de l'angle d'observation le sens de l'interprétation de la culture change. Ce sens est notamment relatif à l'échelle d'observation et au découpage empirique réalisé à une échelle donnée par le chercheur (cf. D. Desjeux. 1996).

6 La question des échelles permet de résoudre une partie des difficultés liées à l'observation de la culture : ce qui est observé a l'échelle macro-sociale et qui permet de repérer les régularités d'une culture, disparaît à l'échelle micro-sociale où les diversités culturelles et comportementales dominent. Les deux sont vrais en même temps et c'est cela qui est troublant, voire difficile à accepter, mais qui pourtant permet de mieux comprendre la portée et les limites de chaque approche culturelle. La théorie des échelles d'observation est une approche anthropologique qui permet de comprendre la diversité des approches, sans faire perdre à chacune son identité, tout en évitant une approche globale "impérialiste», en favorisant les mélanges et tout en montrant qu'il existe des mécanismes universaux à toutes les cultures. Mais toutes ces dimensions ne sont pas saisissables en même temps. En fonction des enquêtes et des problèmes le chercheur saisit plutôt la différence, la stabilité, l'universel ou le changement. En physique Heisenberg a déjà montré qu'on ne peut saisir en même temps deux dimensions, la position et le mouvement d'une particule. Nous nous heurtons au même problème en sciences humaines, au moins de façon analogique par rapport à la physique. 


\section{La recherche des stabilités culturelles dans le monde et dans les organisations}

7 À l'échelle macroscopique, il est recherché les stabilités, celle des aires culturelles, des religions et des valeurs. Ce sont des approches qui privilégient le plus souvent la longue durée historique qui est l'équivalent dans le temps de l'échelle macro-sociale qui elle est synchronique, à un moment donné. Elles sont réductionnistes, et c'est là leur qualité et leur intérêt, car elles saisissent des ensembles très vastes en les simplifiant comme c'est le cas pour les aires culturelles en géopolitique. Elles sont partiellement déterministes ce qui permet de mieux voir les tendances, de gommer les aspérités que l'on observe surtout à l'échelle micro-sociale, et c'est là leur limite : elles ne peuvent pas prendre en compte le côté contingent des situations concrètes. Elles montrent en même temps l'importance des structures dans l'explication des comportements humains, et donc que l'action en société ne se joue pas dans un vide social indéterminé, et en même temps l'impossibilité de prédire l'avenir du fait des effets de contingence et de situation au-delà des effets de structure.

$8 \quad$ Le cas des aires culturelles en est un bon exemple. Il existe un débat sur le nombre d'aires culturelles dans le monde. Leur nombre peut varier d'une dizaine à une trentaine voire à l'infini en fonction des critères de classification choisis. Emmanuel Todd (1983) part d'une hypothèse anthropologique : ce n'est pas la politique qui façonne les valeurs d'une société mais le creuset familial. Ce creuset familial est lui-même organisé par des pratiques et des valeurs qui sont déterminés par les règles de la filiation, de l'alliance et de la résidence. Ainsi la filiation, de qui est-on le descendant, fixe les règles de l'héritage. L'héritage peutêtre égalitaire ou inégalitaire entre les membres de la fratrie. Ces règles varient en fonction du sexe ou de l'âge, notamment le droit d'aînesse. C'est pourquoi pour Emmanuel Todd un héritage inégalitaire semble favoriser la production sociale de valeurs inégalitaires. La valeur accordée à l'autorité et à la liberté paraît liée aux règles de résidence, cohabiter longtemps ou quitter rapidement le toit familial. Une cohabitation longue, comme pour les "Tanguy » aujourd'hui, favoriserait les valeurs autoritaires. La liberté serait liée aux règles de l'alliance, du mariage: en favorisant l'endogamie, se marier dans sa famille, on favorise la dépendance ; l'exogamie, se marier en dehors de sa famille, pousse à s'autonomiser. À partir de ces valeurs il distingue 7 grands modèles politiques basés sur 7 modèles familiaux dont le plus important est celui qu'il appelle le modèle « communautaire exogame », qui privilégie l'autorité et l'égalité dans la famille. Il représente $41 \%$ de la population mondiale et c'est là que se sont développés les systèmes politiques communistes. Pour Emmanuel Todd, c'est la culture familiale qui explique la culture politique et par là les grandes aires culturelles.

D'autre comme Inglehart et Huntington partent de critères religieux pour comprendre la géopolitique mondiale. L'enquête de R. Inglehart, citée par Jean-Claude Ruano-Borbaland (mars 2000, p.18) et qui porte sur les aires religieuses dans le monde, confirme empiriquement, à peu de chose près, la catégorisation de Samuel Huntington en 9 grandes aires culturelles (occidentale, latino-américaine, africaine, islamique, chinoise, hindoue, orthodoxe, bouddhiste et japonaise, 1997, p. 21).

10 Ce qui varie c'est leur interprétation du conflit à venir : pour Huntington cette division du monde en aires culturelles, religieuses et ethniques transcende les clivages politiques et serait source d'une tension de fond entre l'Occident et l'Islam tout particulièrement. Pour 
Inglehart d'après J-C. Ruano la mondialisation semblerait plutôt se traduire sur la longue durée par une plus forte acceptabilité des différences et donc des risques de conflits qui devraient aller en diminuant. Emmanuel Todd (2002) montre aussi qu'un scénario de pacification est plausible, en s'appuyant sur la baisse du taux de natalité associé à l'augmentation du taux de scolarisation pour montrer que l'évolution de ces deux taux va dans le sens d'une plus grande démocratisation et par là d'une fin possible de l'histoire et des conflits, au moins tendanciellement et donc que la thèse de Fukuyama de la fin de l'histoire n'est pas à écarter.

11 Dans les organisations, les socio-anthropologues cherchent à comprendre, toujours à l'échelle macro-sociale, les effets de " programmation culturelle » (Daniel Bollinger, Geert Hofstede 1987). Geert Hofstede montre dans une célèbre enquête statistique, publiée en 1980 en anglais et menée dans 72 filiales IBM dans le monde, que contrairement aux apparences il n'observe pas une convergence des cultures, on dirait aujourd'hui une mondialisation uniformisante, mais au contraire un maintien de la diversité des cultures nationales. Il montre notamment que certains pays comme les pays latins, musulmans et d'Afrique noire valorisent la distance hiérarchique au contraire des pays Scandinaves comme le Danemark. On peut voir ici un bon exemple de régularité macro-culturelle, la valorisation de la distance hiérarchique, vraie comme régularité statistique, mais qui peut tout à fait se révéler non pertinente à l'échelle micro-sociale, celle des interactions, des relations de pouvoir et des effets de situation.

12 L'échelle macro-sociale est aussi celle des permanences comme chez Jack Goody dans $L$ Orient en Occident (1999) livre dans lequel il relativise sur la longue durée, 5000 ans, les différences d'évolution historique entre les cultures orientales et occidentales. Tout particulièrement il critique l'affirmation du « retard chinois » depuis le $18^{\mathrm{e}}$ siècle, en le comparant à la stagnation européenne entre le $4^{\mathrm{e}}$ et le $10^{\mathrm{e}}$ siècle. L'approche par la longue durée relativise la diversité. Elle réduit la diversité des comportements aux grandes représentations symboliques. C'est sa dimension réductrice qui en fait tout son intérêt géopolitique, mais aussi sa limite quand elle veut s'appliquer à l'échelle micro-sociale et au changement hors de tout effet de situation.

\section{Le lien problématique entre effet de culture macro- sociale et effet de situation microsociale}

13 C'est bien le problème auquel s'était heurté Michel Crozier dans les années soixante dans Le phénomène bureaucratique (1963) quand il cherchait à expliquer le lien entre le fonctionnement de la bureaucratie française et des «traits culturels assez bien établis en France » (p. 265) pour faire ressortir que la difficulté à changer des français était liée à leur forte incapacité à coopérer positivement, elle-même liée au système scolaire. À l'école d'après l'américain Pitts, cité par M. Crozier, le seul moment d'action collective est un moment de coopération négatif, celui du chahut contre le professeur. Mais mobiliser la culture française, ici celle du chahut, - même si on sait depuis que la nature du chahut a pu changer en France et devenir anomique - pour expliquer les résistances au changement de l'administration française rend difficile de rendre compte des changements de cette même administration par la suite. Comme l'enquête de Claude Giraud (1987) sur France Télécom l'a montré pour les années quatre vingt, les entreprises publiques ont des capacités à changer. France Télécom a peut-être même trop changé aujourd'hui, mais c'est une autre histoire... 
14 Face à cette difficulté de montrer un lien empirique concret entre la macro-culture statique et les comportements des acteurs face au changement. Michel Crozier abandonnera cette approche culturaliste qui sera reprise plus tard, à nouveau frais, par Philippe d'Iribarne et son équipe, et tout particulièrement par Sylvie Chevrier. Dans son travail sur Le management des équipes interculturelles (2000) elle montre qu'il existe plusieurs jeux stratégiques possibles de gestion de la situation interculturelle entre un modèle diplomatique qui produit une troisième culture, différente des cultures en présence, un modèle hiérarchique qui impose une culture, et un modèle laisser faire. En terme plus stratégique et micro-social, ces exemples montrent qu'entre le macro et le micro il existe un effet de situation, avec des contraintes qui font que la culture peut jouer comme contexte mais pas comme variable explicative indépendante.

15 Il faut donc accepter que certaines observations macro-culturelles soient justes à cette échelle, mais que leur pertinence explicative disparaisse quand on change d'échelle, et donc que le lien entre un modèle explicatif macro-culturel et des pratiques micro-sociales n'est pas mécanique.

16 Les socio-économistes se sont heurtés à une difficulté d'un autre ordre, non plus entre deux échelles mais à la même échelle: comment la culture peut-elle expliquer la croissance et la crise. En Asie du Sud Est avant 1997, la croissance économique et le succès des « cinq dragons » avaient été expliqués par le confucianisme et la morale d'ordre qu'il impliquait dans les entreprises et donc la pression qu'il exerçait sur les salariés. Puis la crise venant, le confucianisme restant, il a bien fallu se rendre à l'évidence que le confucianisme, vu d'un point de vue essentialiste, ne pouvait pas expliquer à la fois le succès et la crise économique, sauf à prendre un point de vue « situationniste » comme le font Peter L. Berger et Hsin-Huang Michael Hsiao (1993). Berger avait déjà montré que la culture ne pouvait pas être appréhendée comme une variable statique (p.11), mais comme une variable stratégique dont la force explicative variait en fonction de la situation historique. La même morale, la même religion peut devenir cause de succès ou d'échec en fonction de la conjoncture. La même religion, le christianisme a pu donner l'Inquisition et les mouvements de libération dans le tiers monde sud américain huit siècles plus tard. Le protestantisme a pu être positif par rapport au développement du capitalisme au $16^{\mathrm{e}}$ siècle, mais le catholicisme a pu lui être plus favorable à d'autres périodes plus récentes comme le montre les économistes pour l'Italie du Nord de la fin du $20^{\mathrm{e}} »$ siècle.

17 La culture possède bien une capacité explicative mais surtout en situation ce qui relativise sa force d'explication. Cela revient à dire que les valeurs, les modèles culturels, les habitus ou les visions du monde ne sont qu'en partie explicatives des comportements humains. D'un côté ils structurent nos comportements, et de l'autre les contraintes matérielles, sociales ou symboliques font que ces modèles culturels ne peuvent s'appliquer tels quels. Ils sont transformés par les effets de situation. C'est une des sources de l'explication de l'écart entre ce qu'on dit, ce qu'on pense et ce qu'on fait. C'est ce que j'observe quand je passe à l'échelle d'observation microsociale. 


\section{À l'échelle microsociale la culture disparaît en partie au profit de l'effet de situation}

En effet, en changeant d'échelle d'observation pour travailler sur les interactions entre des acteurs dans une organisation, un quartier ou un espace domestique, il est plus difficile de distinguer les effets de culture, des effets de situation ou des effets de rapport de pouvoir. C'est pourquoi, à cette échelle la place de la culture comme variable explicative des comportements humains et des différences interculturelles est très ambiguë.

Bien souvent, à cette échelle, je constate que dans une interaction conflictuelle la culture est mobilisée pour éliminer les sources plus conflictuelles du problème que ce soit en déclarant que les américains sont des "grands enfants »- de façon étonnante du reste comme les africains - que les français sont «arrogants» ou que les chinois «ne respectent pas les contrats » et sont "méfiants", au lieu d'élucider des différences de logiques dans la façon de résoudre les problèmes techniques ou de management, les différences d'intérêt ou les rapports de pouvoir pour le contrôle de tel ou tel espace professionnel. L'explication par les valeurs culturelles sensées déterminer les comportements des acteurs avec qui l'on a à travailler, devient une façon de nier l'autre et de masquer les rapports de pouvoir. Mobiliser la culture n'a pas ici une fonction de connaissance. Elle a une fonction de sécurisation: attribuer à l'autre la source de nos problèmes. C'est souvent psychologiquement utile pour supporter une situation interculturelle difficile. Dans ces conditions l'explication culturelle relève de la croyance. L'explication par la culture permet de se libérer dans l'imaginaire, et pourquoi pas, d'une situation réelle de contact entre deux cultures dans laquelle il n'y pas de solution simple.

Cependant l'échelle micro-sociale permet la comparaison entre cultures et pratiques. Elle permet de montrer à la fois qu'il existe des mécanismes généraux voire universels, mais que leurs formes peuvent être particulières en fonction des groupes, des sociétés ou des époques. Ainsi, dans la plupart des sociétés aujourd'hui il existe un jeu de l'oral et de l'écrit dans le management des organisations, ou encore il y a partout des rapports de pouvoir, ou encore chaque société accorde de l'importance aux jeux de face comme l'a montré Goffman pour les USA, en s'inspirant de la Chine semble-t-il, Zheng Li Hua pour les chinois. Je l'ai retrouvé sous d'autres noms ailleurs, avec le nif pour les arabes ou l'expression «faire perdre la face » en France.

21 Ce qui peut varier c'est l'importance accordé aux jeux de face, à l'oral ou à l'écrit, aux enjeux de pouvoir, à la honte en fonction des domaines d'application. Comme le montre Zheng Li hua (2002), les français écrivent là où les chinois ne s'y attendent pas. Ils font des notes de travail bien trop longues là où une interaction verbale suffirait et serait plus efficace qu'un texte écrit.

C'est aussi à cette échelle, qu'en comparant des itinéraires de pratiques de consommation alimentaire, à une échelle très micro-sociale, entre la France, les USA, la Chine et le Danemark je me suis rendu compte que toutes les cultures accordaient de l'importance au fait de se nourrir et que même les américains pouvaient parler longtemps à table des plats de leur région d'origine comme les français, mais qu'en fonction des cultures il était accordé plus ou moins d'importance au temps de cuisine, au temps de repas, et aux restes 
(cf. sur les itinéraires en photos: www.argonautes.fr; et Dominique Desjeux, Isabelle Garbuau-Moussaoui, Elise Palomares (éds.). 2002)

De même si je reviens à un niveau plus macro-social, il semble que dans toutes les sociétés on trouve un principe explicatif de la vie qui dépasse les volontés individuelles, qu'il s'appelle le fatum chez les grecs, le mektoub chez les arabes, le Shi chez les chinois, le pouvoir magico-religieux lié à la sorcellerie en Afrique, la grâce chez les chrétiens ou le déterminisme scientifique dans les sociétés occidentales. Ce principe peut être immanent ou transcendant. Chacun peut y adhérer ou le contester, et c'est bien là que l'analyse des différences culturelles redevient pertinente à la fois pour rechercher les invariants anthropologiques et les variations culturelles infinies, notamment au niveau macroculturel.

\section{Conclusion}

L'outil des échelles d'observation permet de faire apparaître une confusion fréquente entre deux échelles, l'une macro-sociale qui permet d'observer les grandes variables et les grandes stabilités culturelles qui structurent le champ de l'action en société, mais avant d'être passé au tamis de l'effet de situation et des contraintes qui font que les acteurs ne se comportent pas de fait comme le voudraient les valeurs décrites grâce à une approche macro-sociale; l'autre microsociologique à laquelle les valeurs et la culture disparaissent en partie au profit de l'effet de situation et des contraintes de l'action. L'important est de retenir l'ambivalence de l'outil culturel et la tension qui sous tend son usage. La culture peut autant être une clé pour mieux fonctionner avec l'autre qu'un moyen de le nier.

\section{BIBLIOGRAPHIE}

BERGER P. L.. HSIAO H-H. M., In Search of an East Asian Development Model. New Brunswick : Transaction Publishers. 1993. 243 p. (1 édition 1987)

BOLINGER D.. HOFSTEDE G., Les différences culturelles dans le management. Paris : Les éditions d'organisation. 1987. 268 p. (1 $1^{\mathrm{e}}$ édition anglaise 1980)

CHEVRIER Sylvie. Le management des équipes interculturelles. Paris : PUF, 2000. 211 p.

CROZIER M., Le phénomène bureaucratique. Paris : Le Seuil. 1963. 382 p.

DESJEUX D.. GARABUAU-MOUSSAOUI I., PALOMARES E., (éds.). Alimentations contemporaines. Paris : L'Harmattan, 2002. 397 p.

DESJEUX D., (en collaboration avec Sophie Taponier), Le sens de l'autre. Stratégies, réseaux et cultures en situation interculturelle. Paris : Unesco. 169 p. (1999. l'Harmattan. $3^{\mathrm{e}}$ édition)

DESJEUX D., « Tiens bon le concept, j'enlève l'échelle... d'observation », UTINAM nº 20. 1996, p. $15-44$ 
DORTIER J-F., (éd.). La communication appliquée aux organisations et à la formation. Paris : Démos. $1998.173 \mathrm{p}$.

FAURE G., RUBIN J., (éds.). Culture and Négociation. Londres : Sage. 1983.264 p.

GIRAUD C. Bureaucratie et changement. Paris : L'Harmattan. 1987. 262 p.

GOODY J., L 'Orient en Occident. Paris : Le Seuil. 1999. 394 p.

HALL E. T., Guide du comportement dans les affaires internationales. Paris : Seuil, 1990, 224 p.

HASON V. D., Carnage et culture. Les grandes batailles qui ont fait l'occident. Paris : Flammarion. 2002. $600 \mathrm{p}$.

HUNTINGTON S., P., Le choc des civilisations. Paris : O. Jacob. 1997. 402 p. (1 ${ }^{\text {re }}$ édition américaine 1996)

IRIBARNE (d') P.. La logique de l'honneur. Paris : Le Seuil, 1989. 280 p.

IRIBARNE (d')P., HENRY A., SEGAL J-P., CHEVRIER S.. Tatjana GLOBOKAR T., Cultures et mondialisation. Paris : Le Seuil. 1998. 355 p.

KYMLICKA W.. MESURE S., (éds.). Comprendre les identités culturelles. Revue de philosophie et de sciences sociales. $\mathrm{n}^{\circ} 12000.422 \mathrm{p}$.

LACORNE D. La crise de l'identité américaine. Paris : Fayard. 1997.

PONCELET M., Une utopie post-tiersmondisme. La dimension culturelle du développement. Paris :

L'Harmattan, 1994. 366 p.

RUANO-BORBALLAN J-C. Valeurs et cultures : allons-nous devenir post modernes ?, Sciences

Humaines $\mathrm{n}^{\circ}$ 103. $\mathrm{p}$ 16-20

TODD E.. Après l'empire. Essai sur la décomposition du système américain. Paris : Gallimard, 2002.

$233 \mathrm{p}$.

TODD E., La troisième planète. Structures familiales et systèmes idéologiques. Paris : Le seuil, 1983. $252 \mathrm{p}$.

ZHENG L-H., DESJEUX Dominique, (éds.). Comment les chinois voient les européens. Paris : PUF, 2003

ZHENG L-H., DESJEUX Dominique, (éds.). Entreprises et vie quotidienne en Chine. Paris : L'Harmattan. 2002. $301 \mathrm{p}$.

www.argonautes.fr : présentation de la méthode des itinéraires avec des photos et des articles.

\section{RÉSUMÉS}

L'usage social de la dimension culturelle peut autant servir à nier l'autre qu'à le comprendre. Cela dépend en partie des échelles d'observation à laquelle acteurs et observateurs se situent. L'échelle macro-culturelle permet de comprendre les grandes permanences, mais est de peu d'utilité dans l'action. Elle peut aussi transformer la culture en instrument de domination et d'exclusion de l'autre au nom de valeurs abstraites présentées comme incompatibles. L'échelle micro-sociale, tout en prenant en compte la différence culturelle grâce aux modèles de résolution des problèmes, la relativise au profit de l'effet de situation, de réseaux et de stratégie d'acteurs.

The social use of the cultural dimension can serve to negate others as well to understand them. It depends to a certain extent on which scale of observation actors or observers are using. The macro-cultural scale may help explain more permanent cultural structures but it is not very 
useful with actions. It can also transform culture into an instrument of domination and exclusion of others by means of abstract values that are presented as incompatible. The micro-social scale, which deals with cultural differences by means of problem-solving models, puts them into perspective with the effects of situation, network, and actor strategy

INDEX

Mots-clés : culture, échelles d'observation, réseau, modèle de résolution des problèmes, situation.

\section{AUTEUR}

\section{DOMINIQUE DESJEUX}

Dominique Desjeux est professeur d'anthropologie sociale et culturelle à la Sorbonne université Paris 5. Depuis 1969 il a mené de nombreuses enquêtes en France et en Europe, en Afrique, en Asie et aux USA sur la consommation, les prises de décision en organisation et la dimension interculturelle des innovations professionnelles et domestiques. 